\title{
Numerical simulation of phase change and contact lines on unstructured grids: towards simulation of nucleate boiling
}

\author{
Savinien Pertant ${ }^{* 1}$, Giovanni Ghigliotti ${ }^{1}$, Guillaume Balarac ${ }^{1,2}$, Guillaume Sahut $^{1}$ \\ ${ }^{1}$ Univ. Grenoble Alpes, CNRS, Grenoble INP, LEGI, 38000 Grenoble, France \\ ${ }^{2}$ Institut Universitaire de France (IUF), Paris, France \\ ${ }^{*}$ Corresponding author email: savinien.pertant@univ-grenoble-alpes.fr
}

\begin{abstract}
In this work, various simulations of boiling on unstructured grids are performed. The methodology introduced in [14] is employed. We show that the use of the conservative level-set instead of the standard signed distance level-set increases the method accuracy. In particular, the bubble rise and growth is simulated with dynamic mesh adaptation to keep high resolution around the interface with moderate number of cells. Finally, film boiling with bubble formation is simulated in 2D. This case marks a milestone towards the simulation of nucleate boiling, where in addition a liquid-vapor-solid contact has to be simulated. Furthermore, simulations of contact lines without phase change are performed to assess a contact angle imposition method representing the surface wettability. The methodology is validated on a canonical test case and reproduces accurately the drop impact on a superhydrophobic cone. On-going work is then devoted to coupled numerical methods for boiling with these new developments.
\end{abstract}

\section{Keywords}

Boiling, Unstructured mesh, Conservative level-set, Contact angle, Drop impact.

\section{Introduction}

Two-phase flows are found in various natural phenomena and industrial applications. More specifically, boiling is widely used for heat exchanges in nuclear plants or in refrigerating machines. Most of the applications are interested in nucleate boiling, a regime for which vapor bubbles are nucleated on the heated surface and detach afterwards due to buoyancy effect. Nucleate boiling implies several complex physical phenomena. First, heat conduction induces phase change at the liquid-vapor interface, creating physical discontinuities. Second, three phases coexist at the intersection between the liquid-vapor interface and the solid surface. This closed curve named contact line is characterized by a contact angle, representing the surface wettability. Numerical simulation of nucleate boiling requires to compute accurately the amount of liquid transformed into vapor and must take into account the surface wettability effects which can be determining on the global heat transfer. Based on the methodology devoted to simulate phase change on unstructured meshes described in [14], this article presents several cases of boiling in the aim of simulating nucleate boiling. In addition, simulations of contact lines without phase change are performed to show the solver capacity to take surface wettability effects into account, even on complex geometries.

\section{Governing equations and interface conditions}

The flow is described by the incompressible Navier-Stokes equations. For any field $A$, we define the interface jump operator $[A]_{\Gamma}=A_{l, \Gamma}-A_{g, \Gamma}$, where the subscript $l$ stands for the liquid phase and $g$ for the gaseous phase. The mass conservation reads in each phase $\nabla \cdot \mathbf{u}=0$, where $\mathbf{u}$ is the fluid velocity. If phase change occurs, the velocity field is discontinuous at interface $\Gamma$ and must satisfy the condition

$$
[\mathbf{u}]_{\Gamma}=\dot{m}\left[\frac{1}{\rho}\right]_{\Gamma} \mathbf{n}
$$


where $\dot{m}$ is the local mass transfer rate through the interface, $\rho$ the density and $\mathbf{n}$ the interface normal vector. Assuming uniform density in each phase, the momentum conservation reads

$$
\frac{\partial \mathbf{u}}{\partial t}+\nabla \cdot(\mathbf{u} \otimes \mathbf{u})=-\frac{\nabla P}{\rho}+\frac{1}{\rho} \nabla \cdot\left(\mu\left(\nabla \mathbf{u}+(\nabla \mathbf{u})^{T}\right)\right)+\mathbf{g},
$$

where $P$ is the pressure, $\mathbf{g}$ the gravitational acceleration and $\mu$ the dynamic viscosity of the considered phase. The pressure discontinuity at the interface is defined by

$$
[P]_{\Gamma}=\sigma \kappa+\left[\mu \mathbf{n} \cdot\left(\nabla \mathbf{u}+(\nabla \mathbf{u})^{\top}\right) \mathbf{n}\right]_{\Gamma}-\dot{m}^{2}\left[\frac{1}{\rho}\right]_{\Gamma},
$$

where $\sigma$ is the surface tension coefficient and $\kappa$ the local interface curvature. The pressure is thus discontinuous even without phase change, contrary to the velocity.

In each phase, energy conservation is expressed with the temperature and reads

$$
\frac{\partial T}{\partial t}+\mathbf{u} \cdot \nabla T=\frac{1}{\rho c_{\mathrm{p}}} \nabla \cdot(\lambda \nabla T)
$$

where $T$ is the temperature, $c_{\mathrm{p}}$ is the heat capacity at constant pressure and $\lambda$ is the thermal conductivity of the considered phase. When phase change is considered, the heat flux at interface is not entirely transmitted from one phase to the other and one has the following power balance $[-\lambda \nabla T \cdot \mathbf{n}]_{\Gamma}=\dot{m} L_{v}$, where $L_{v}$ is the latent heat of vaporization. This is the definition of the local mass transfer rate $\dot{m}$. Moreover, since we focus on boiling problems, the interface temperature is set to the saturation temperature, i.e. $T_{\Gamma}=T_{\text {sat }}$. The Navier-Stokes equation and the heat equation are coupled through the jump conditions Eq. (1) and Eq. (3).

\section{Liquid-gas interface capturing method: conservative level-set}

The liquid-gas interface is captured by an improved level-set method, the conservative levelset [12]. The $\psi=0.5$ isocontour of the function $\psi=0.5(\tanh (\phi /(2 \varepsilon))+1)$ represents the interface, where $\phi= \pm\left|\mathbf{x}-\mathbf{x}_{\Gamma}\right|$ is the signed distance function to the interface (positive in the liquid) and $2 \varepsilon$ is the profile thickness. Using the interface velocity $\mathbf{u}_{\Gamma}=\mathbf{u}_{g}-\dot{m} / \rho_{g} \mathbf{n}$, the level-set is advected with an equation derived in [13]

$$
\frac{\partial \psi}{\partial t}+\nabla \cdot\left(\mathbf{u}_{g} \psi\right)=\frac{\dot{m}}{\rho_{g}} \mathbf{n} \cdot \nabla \psi+\psi \nabla \cdot \mathbf{u}_{g} .
$$

The right-hand side vanishes when there is no phase change. The second term of the righthand side takes into account the fact that the divergence-free condition is not necessarily respected in the liquid phase and close to the interface [13].

In this work, the hyperbolic tangent profile is reshaped with the re-initialization equation [3]

$$
\frac{\partial \psi}{\partial \tau}=\nabla \cdot\left(\frac{1}{4 \cosh ^{2}\left(\frac{\phi_{\mathrm{map}}}{2 \varepsilon}\right)}\left(\nabla \phi_{\mathrm{map}} \cdot \mathbf{n}-1\right) \mathbf{n}\right),
$$

which is solved in pseudo-time $\tau$ until steady state, and where $\phi_{\text {map }}=\varepsilon \ln (\psi /(1-\psi))$ is a signed distance function mapped from the conservative level-set. The interface normal vector is defined as $\mathbf{n}=\nabla \phi /\|\nabla \phi\|$.

\section{Numerical method}

The simulations are performed with the code YALES2, a library of finite-volume node-centered low Mach number solvers [11]. The Navier-Stokes equations are solved with a standard projection method [4]. The numerical method to perform boiling simulations is presented in [14]. The Ghost Fluid Method [5] is used, enabling a sharp treatment of the discontinuities at the interface. In [14], the standard distance level-set was used, whereas the conservative level-set 
is employed in the present study. The level-set re-initialization algorithm was adapted to unstructured meshes by [8]. Another slight difference resides in the velocity extensions across the interface. As a matter of fact, the velocity discontinuity at the interface given by Eq. (1) requires velocity extensions to compute the velocity derivatives close to the interface. In [14], the velocity is extended constantly across the interface with the method developed by [1]. The equation $\partial \mathbf{u}_{i} / \partial \tau+\nabla \mathbf{u}_{i} \cdot \mathbf{n}=0$ is solved in pseudo-time $\tau$ until steady state, with the subscript $i$ representing the phase. At steady state, this equation imposes that the velocity derivative in the direction normal to the interface is null. In this study we use instead the method employed by [16] for simulation of boiling flows on cartesian grids. The ghost velocities are defined as

$$
\begin{aligned}
& \mathbf{u}_{l}^{G}=\mathbf{u}_{g}+\dot{m}\left[\frac{1}{\rho}\right]_{\Gamma} \mathbf{n} \quad \text { if } \psi<0, \\
& \mathbf{u}_{g}^{G}=\mathbf{u}_{l}-\dot{m}\left[\frac{1}{\rho}\right]_{\Gamma} \mathbf{n} \quad \text { if } \psi>0 .
\end{aligned}
$$

This ensures that the velocity jump is constant in the direction normal to the interface.

In addition, for contact line simulations, the contact angle is imposed thanks to a curvature modification at contact line. Following [17], a sub-grid scale curvature $\kappa_{S G S}$ is added to the interface curvature $\kappa$ at contact line, such that

$$
\kappa_{C L}=\kappa+\kappa_{S G S}=\kappa-\frac{\mathbf{n} \cdot \mathbf{n}_{\text {wall }}+\cos \left(\theta_{\mathrm{eq}}\right)}{h},
$$

where $\mathbf{n}_{\text {wall }}$ is the wall normal vector, $\theta_{\text {eq }}$ the imposed contact angle and $h$ half of the cell size at wall. The additional surface tension force at contact line is thus proportional to the difference between measured and imposed contact angles. Furthermore, a singularity at contact line appears when a no-slip condition is used at wall [7]. To alleviate this problem, a Navier slip condition is used, defined by

$$
\mathbf{u}_{\|}=\left.\lambda \frac{\partial \mathbf{u}_{\|}}{\partial y}\right|_{\text {wall }},
$$

where $\mathbf{u}_{\|}$is the wall tangential velocity, $y$ the wall normal coordinate and $\lambda$ a slip length. This allows the contact line movement on the wall.

\section{Boiling simulations without contact line}

The solver is first assessed on various test cases with phase change.

\section{Growth of a 3D static bubble in a superheated liquid}

A standard test case to assess a boiling solver consists in a vapor bubble growth in a superheated liquid. The vapor phase is initially at saturation temperature while the temperature profile in the liquid is radially symmetric. The full analytical solution has been derived by [15] and is recalled in [13]. The gravity being neglected, the bubble remains spherical and its center of mass does not move. The simulation is initialized at a time $t_{0}$ with the analytical solution. The theoretical radius is given at any time $t>t_{0}$ by $R_{\mathrm{th}}(t)=2 \beta \sqrt{\alpha_{l} t}$, where $\alpha_{l}=\lambda_{l} /\left(\rho_{l} c_{\mathrm{p}, l}\right)$ is the liquid thermal diffusivity. The parameter $\beta$ is found from an implicit equation as detailed in [14]. It depends on the Jakob number, defined as $\mathrm{Ja}=\rho_{l} c_{\mathrm{p}, l}\left(T_{\infty}-T_{\mathrm{sat}}\right) /\left(\rho_{g} L_{v}\right)$, where $T_{\infty}$ is the temperature in the liquid far away from the interface. The following simulations are performed for $\mathrm{Ja}=3$. The physical parameters representing liquid water and vapor are the same as in [14] and are recalled in Table 1. The heat capacity at constant pressure of the liquid has been purposely decreased to reduce computational time, as explained in [14]. The simulations are performed until $t_{f}=4 t_{0}$, meaning that $R_{\mathrm{th}}\left(t_{f}\right)=2 R_{\mathrm{th}}\left(t_{0}\right)=2 R_{0}$, with $R_{0}=0.001 \mathrm{~m}$. Outlet conditions are applied on the domain boundaries, allowing the liquid to exit the domain instead of being compressed. The present case consists in the extension of the work performed in [14] with signed distance function to the conservative level-set. The relative errors on the computed 
Table 1. Physical properties for simulation of the growth of a 3D bubble in a superheated liquid.

\begin{tabular}{cccccccc}
\hline Phase & $\begin{array}{c}\rho \\
{\left[\mathrm{kg} \mathrm{m}^{-3}\right]}\end{array}$ & $\begin{array}{c}\mu \\
{\left[\mathrm{kg} \mathrm{s}^{-1} \mathrm{~m}^{-1}\right]}\end{array}$ & $\begin{array}{c}c_{\mathrm{p}} \\
{\left[\mathrm{J} \mathrm{kg}^{-1} \mathrm{~K}^{-1}\right]}\end{array}$ & $\begin{array}{c}\lambda \\
{\left[\mathrm{W} \mathrm{m}^{-1} \mathrm{~K}^{-1}\right]}\end{array}$ & $\begin{array}{c}L_{v} \\
{\left[\mathrm{~J} \mathrm{~kg}^{-1}\right]}\end{array}$ & $\begin{array}{c}\sigma \\
{\left[\mathrm{N} \mathrm{m}^{-1}\right]}\end{array}$ & $\begin{array}{c}T_{\text {sat }} \\
{[\mathrm{K}]}\end{array}$ \\
\hline$l$ & 958 & $2.82 \times 10^{-4}$ & 42.16 & 0.6 & & \\
$g$ & 0.59 & $1.23 \times 10^{-6}$ & 2034 & 0.026 & $2.257 \times 10^{6}$ & $5.9 \times 10^{-2}$ & 373 \\
\hline
\end{tabular}

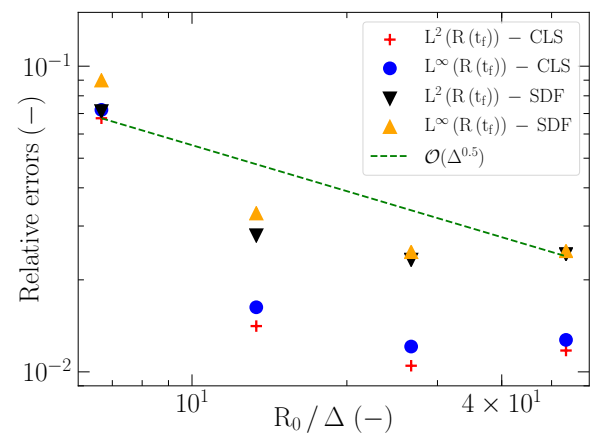

Figure 1. Relative errors on bubble radius for 3D bubble growth in a superheated liquid versus the number of cells along the bubble initial radius. CLS stands for conservative level-set and SDF stands for signed distance function. The standard $\mathrm{L}^{2}$ and $\mathrm{L}^{\infty}$ norm are used.

radius at $t_{f}$ are presented on Fig. 1 for both the signed distance function and the conservative level-set, for four different cell sizes. For both level-set methods, the error converges initially with mesh refinement before reaching a plateau. As previously observed by $[16,14]$ for $\mathrm{Ja}=3$, the error reaches a minimum value when the thermal boundary layer is sufficiently resolved. This error, about $1 \%$ for conservative level-set, is considered satisfactory in this study. Also, it can be seen that the use of conservative level-set leads to lower errors, especially for fine meshes. Therefore we only use the conservative level-set in the next sections.

\section{Rising of a 3D growing bubble}

We now simulate the rising of a growing bubble in a superheated liquid. This study has been recently performed on a $2 \mathrm{D}$ axisymmetric cartesian mesh by [16]. The configuration is the same as in the previous section except that gravity $\left(g=9.81 \mathrm{~m} \mathrm{~s}^{-1}\right)$ is taken into account. The physical properties are identical as in Table 1, only the heat capacity at constant pressure of the liquid is set back to its standard value $c_{\mathrm{p}, l}=4216 \mathrm{~J} \mathrm{~kg}^{-1} \mathrm{~K}^{-1}$ and the surface tension is lowered to $\sigma=0.001 \mathrm{~N} \mathrm{~m}^{-1}$. The temperature is initialized as in the previous section with $\mathrm{Ja}=3$ and the initial bubble radius is $R_{0}=0.0001 \mathrm{~m}$. The simulations are performed until the time $t_{f}=18 t_{0}$. The mesh is always kept fine around the interface thanks to dynamic mesh adaptation, thus limiting the simulations cost [10]. The bubble grows and rises due to buoyancy. It does not remain spherical because of the low surface tension coefficient, as illustrated on Fig. 2a. A thermal plume below the bubble can be observed. The temperature gradients being lower in this area, the mass transfer rate is lower than above the bubble where the thermal boundary layer is thinner. As in [16], we plot the evolution of the Nusselt number against the square root of the Peclet number on Fig. $2 b$ for two cell sizes. The Nusselt number is defined as

$$
\mathrm{Nu}=\rho_{g} L_{v} \frac{\mathrm{d} R}{\mathrm{~d} t} \frac{2 R}{\lambda_{l}\left(T_{\infty}-T_{\mathrm{sat}}\right)}
$$

where $R$ is the bubble equivalent radius. The Peclet number is defined as $\mathrm{Pe}=2 \rho_{l} c_{\mathrm{p}, l} V_{b} R / \lambda_{l}$, with $V_{b}$ the average bubble ascending velocity.

A slight difference in the Nusselt number can be observed between the two cell sizes, the finest mesh being closer to the results of [16]. Even if the initial and final values are close to the 


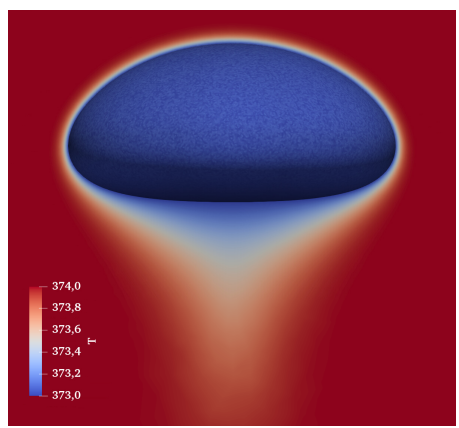

(a)

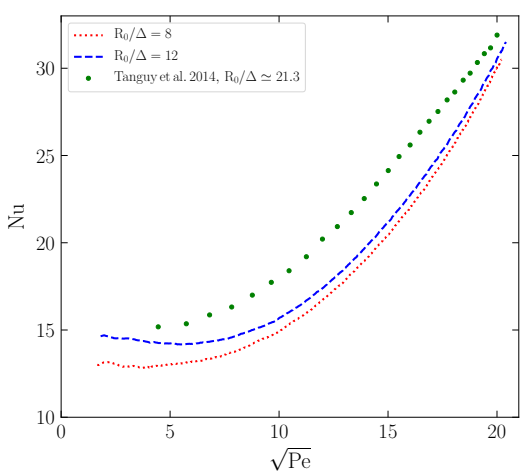

(b)

Figure 2. (a) Rising bubble at final time together with midplane, both colored by temperature. (b) Nusselt number evolution versus square root of the Peclet number square.

reference, the curve shape is however quite different from the one of [16]. It may be explained by the cell size, which can be much smaller in the axisymmetric case with limited cost.

\section{D film boiling}

Film boiling is a pool boiling regime such that the heated wall is covered by a vapor layer. The absence of contact line makes it numerically less complex than nucleate boiling. In [9], the authors simulate 2D film boiling of saturated water at near critical pressure on a cartesian grid. We reproduce this case on unstructured grids here. The physical properties are recalled in Table 2 . The interface is initialized at the location $y=\lambda_{d} / 128\left(4+\cos \left(2 \pi x / \lambda_{d}\right)\right)$ with

Table 2. Physical properties of saturated water at near critical pressure for $2 \mathrm{D}$ film boiling.

\begin{tabular}{cccccccc}
\hline Phase & $\begin{array}{c}\rho \\
{\left[\mathrm{kg} \mathrm{m}^{-3}\right]}\end{array}$ & $\begin{array}{c}\mu \\
{\left[\mathrm{kg} \mathrm{s}^{-1} \mathrm{~m}^{-1}\right]}\end{array}$ & $\begin{array}{c}c_{\mathrm{p}} \\
{\left[\mathrm{J} \mathrm{kg}^{-1} \mathrm{~K}^{-1}\right]}\end{array}$ & $\begin{array}{c}\lambda \\
{\left[\mathrm{W} \mathrm{m}^{-1} \mathrm{~K}^{-1}\right]}\end{array}$ & $\begin{array}{c}L_{v} \\
{\left[\mathrm{~J} \mathrm{~kg}^{-1}\right]}\end{array}$ & $\begin{array}{c}\sigma \\
{\left[\mathrm{N} \mathrm{m}^{-1}\right]}\end{array}$ & $\begin{array}{c}T_{\text {sat }} \\
{[\mathrm{K}]}\end{array}$ \\
\hline$l$ & 402.4 & $4.67 \times 10^{-5}$ & $2.18 \times 10^{5}$ & 0.545 & & \\
$g$ & 242.7 & $3.238 \times 10^{-5}$ & $3.52 \times 10^{5}$ & 0.538 & $2.764 \times 10^{5}$ & $7 \times 10^{-5}$ & 646 \\
\hline
\end{tabular}

$\lambda_{d}=2 \pi \sqrt{3 \sigma /\left(g\left(\rho_{l}-\rho_{g}\right)\right)}$, which is the most unstable Taylor wavelength. This forces the bubble creation at the domain center on the crest of the interface. The computational domain is a square box with a side length $\lambda_{d}$ and periodic conditions are applied on the lateral boundaries. The bottom wall temperature is set to $T_{\text {wall }}=T_{\text {sat }}+5 \mathrm{~K}$. The liquid is initialized at saturation temperature and the vapor temperature decreases linearly from $T_{\text {wall }}$ at the wall to $T_{\text {sat }}$ at the interface. First, a mesh independence study is performed. The cell size has to be small enough to resolve the thermal boundary layer which can be very thin. Four meshes such that $\lambda_{d} / \Delta=100,200,400,800$ are tested. The interface positions are displayed on Fig. 3a. For the coarsest mesh, the bubble formation is too fast while the two finest meshes show similar results. As a matter of fact, if not enough cells are present in the thermal boundary layer, the temperature gradient is not correctly computed, which impacts the mass transfer rate and temperature transport, leading to erroneous overall dynamics. The qualitative behaviour of the film is now studied for the finest grid, using dynamic mesh adaptation. The interface position and the temperature field are shown on Fig. 3b and compared to the results of [9], reproduced on Fig. 3c. The bubble shape is similar between both studies. As previously observed by $[6,18]$, the bubble does not detach from the vapor film. Instead, a long and thin stem presenting low temperature gradients connects the bubble to the film. This behaviour is expected since there is no radial curvature in $2 \mathrm{D}$, which would be responsible for the bubble pinch-off in 3D. The stem being straight, the surface tension effects are negligible. It can be reasonable to assume that 


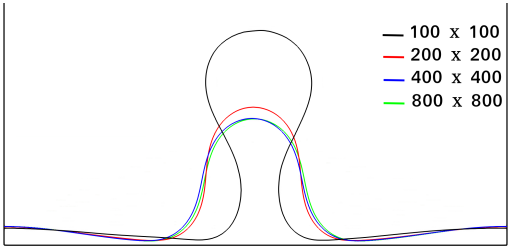

(a)

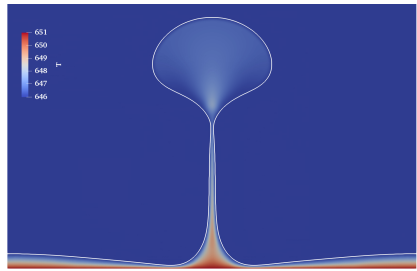

(b)

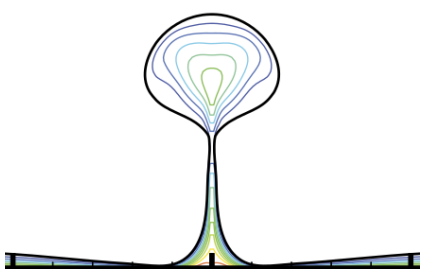

(c)

Figure 3. (a) Interface positions for 2D film boiling at time $t=0.28 \mathrm{~s}$ for four grid resolutions. (b) Interface position (white) for 2D film boiling at time $t=0.338 \mathrm{~s}$ with temperature field. (c) Interface position (black) for 2D film boiling at time $t=0.338 \mathrm{~s}$, results of [9].

the pinch-off mechanism observed in some 2D film boiling studies is due to grid effects.

\section{Contact line simulations without phase change}

The solver ability to simulate two-phase flows with contact lines, without phase change, is assessed in this section.

\section{Equilibrium shape of a 2D drop on a flat wall}

To assess the contact angle imposition method, a standard 2D test case is performed. A steady semicircular drop is initialized on a flat wall with an initial $90^{\circ}$ contact angle. The contact points move in order to respect the imposed contact angle which is different from the initial contact angle. The equilibrium shape of the drop is a circular cap since gravity is null. The radius $R$, the spreading length $L$ and the height $e$ of the equilibrium interface in function of the initial radius $R_{0}$ and the imposed contact angle $\theta_{\text {eq }}$ (in the liquid) are given by

$$
\begin{aligned}
R & =R_{0} \sqrt{\frac{\pi}{2\left(\theta_{\mathrm{eq}}-\sin \left(\theta_{\mathrm{eq}}\right) \cos \left(\theta_{\mathrm{eq}}\right)\right)},} \\
L & =2 R \sin \left(\theta_{\mathrm{eq}}\right), \\
e & =R\left(1-\cos \left(\theta_{\mathrm{eq}}\right)\right) .
\end{aligned}
$$

The physical parameters are taken from [17] and summed up in Table 3 . The simulation is

Table 3. Parameters for 2D drop equilibrium on a flat wall.

\begin{tabular}{ccccc}
\hline Phase & $\begin{array}{c}\rho \\
{\left[\mathrm{kg} \mathrm{m}^{-3}\right]}\end{array}$ & $\begin{array}{c}\mu \\
{\left[\mathrm{kg} \mathrm{s}^{-1} \mathrm{~m}^{-1}\right]}\end{array}$ & $\begin{array}{c}\sigma \\
{\left[\mathrm{N} \mathrm{m}^{-1}\right]}\end{array}$ & $\begin{array}{c}R_{0} \\
{[\mathrm{~m}]}\end{array}$ \\
\hline$l$ & 1000 & $10^{-2}$ & $10^{-5}$ & 0.01 \\
$g$ & 1 & $10^{-5}$ & & \\
\hline
\end{tabular}

performed for various imposed angles ranging from $10^{\circ}$ to $170^{\circ}$, on an unstructured mesh such that $R_{0} / \Delta=20$. In this particular case, a free-slip condition is used at the wall in order to assess only the contact angle imposition method. The measured normalized spreading lengths and heights are plotted on Fig. 4 as a function of the imposed contact angle and are compared to the theoretical ones. The results show a rather good agreement with the analytical solution. For the lowest contact angle, the computed spreading length differs slightly from the analytical solution. This could be explained by the weakness of the capillary forces near equilibrium for 


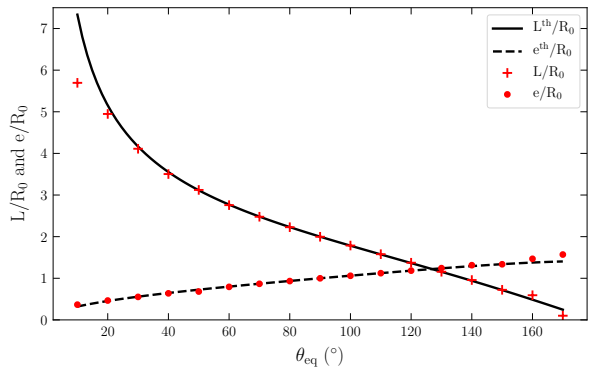

Figure 4. Normalized spreading length $L / R_{0}$ and normalized height $e / R_{0}$ at equilibrium as a function of the imposed contact angle $\theta$ eq.

very low contact angles, competing with numerical artefacts of level-set re-initialization and spurious currents.

\section{Drop impact on a superhydrophobic cone}

The contact angle imposition method being validated, we now simulate the drop impact on a cone. We reproduce the experiments of [2] where a drop of water bounces on a superhydrophobic cone. The drop of radius $R_{0}$ has an initial downward velocity $V_{0}$ and the cone shape is defined by its semi-angle $\alpha$. The gravity is oriented along the cone axis towards the cone basis and the drop bottom is initially at a distance $R_{0} / 4$ from the cone tip. The physical parameters are given in Table 4. The simulations are performed on an unstructured mesh such

Table 4. Parameters for 3D drop impact on a superhydrophobic cone.

\begin{tabular}{cccccc}
\hline Phase & $\begin{array}{c}\rho \\
{\left[\mathrm{kg} \mathrm{m}^{-3}\right]}\end{array}$ & $\begin{array}{c}\mu \\
{\left[\mathrm{kg} \mathrm{s}^{-1} \mathrm{~m}^{-1}\right]}\end{array}$ & $\begin{array}{c}\sigma \\
{\left[\mathrm{N} \mathrm{m}^{-1}\right]}\end{array}$ & $\begin{array}{c}R_{0} \\
{[\mathrm{~m}]}\end{array}$ & $\begin{array}{c}\theta_{\text {eq }} \\
{[-]}\end{array}$ \\
\hline$l$ & 1000 & $10^{-3}$ & 0.072 & 0.0013 & $163^{\circ}$ \\
\hline$g$ & 1 & $1.85 \times 10^{-5}$ & & & \\
\hline
\end{tabular}

that $R_{0} / \Delta=15$. The first case, with an initial velocity $V_{0}=0.23 \mathrm{~m} \mathrm{~s}^{-1}$ and a cone semi-angle $\alpha=50^{\circ}$ is represented on Fig. 5. The second case, with $V_{0}=0.35 \mathrm{~m} \mathrm{~s}^{-1}$ and $\alpha=30^{\circ}$ is represented on Fig. 6 . The snapshots are taken at the same time for the experiments and our simulations. In the first case, the drop spreads on the cone top before bouncing but is not

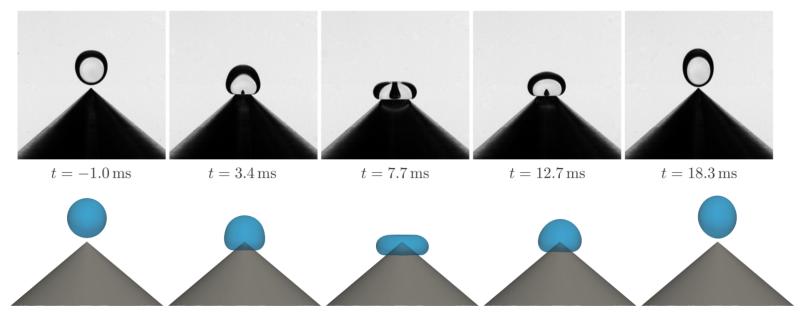

Figure 5. Drop impact $\left(V_{0}=0.23 \mathrm{~m} \mathrm{~s}^{-1}\right)$ on a cone with $\alpha=50^{\circ}$. Top: results from [2].

pierced, while in the second one the drop forms a torus before being repelled. For both cases, a good qualitative agreement with the experiments is found. We find the same bounce times as in the experiments.

\section{Conclusions}

Simulations of boiling on unstructured meshes have been performed successfully in this article. Contrary to [14], the interface is captured with a conservative level-set method, known to alleviate mass conservation issues. This improves the method accuracy on a canonical 3D test case. The employed methodology is able to take into account buoyancy effects, interface 


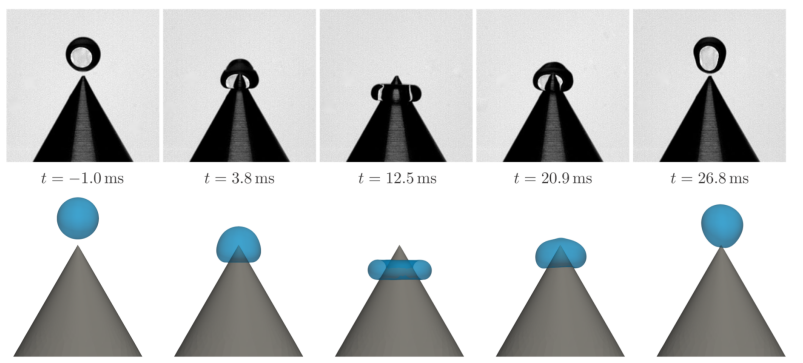

Figure 6. Drop impact $\left(V_{0}=0.35 \mathrm{~m} \mathrm{~s}^{-1}\right)$ on a cone with $\alpha=30^{\circ}$. Top: results from [2].

deformation and thin thermal boundary layers. Dynamic mesh adaptation allows to simulate 3D configurations without a tremendous number of cells. Film boiling is simulated with creation of a bubble from a vapor film. On the other hand, simulations of contact lines are performed in 2D and 3D. Even if further work is needed to couple phase change with contact lines, in particular to deal with the thermal singularity at contact line, these promising results let the authors hope to simulate nucleate boiling with surface wettability effects in a near future.

\section{Acknowledgements}

This work has been supported by the French Ministry of Higher Education, Research and Innovation. The GENCI (Grant 2A00611) and the third edition of the Extreme CFD Workshop (https://ecfd.coria-cfd.fr/) are acknowledged. The authors would like to thank Vincent Moureau, Ghislain Lartigue and Pierre Bénard for providing the code YALES2. Pierre Chantelot is acknowledged for giving the parameters to reproduce drop-cone impact experiments.

\section{References}

[1] Aslam, T.D., 2004, Journal of Computational Physics, 193, pp. 349-355.

[2] Chantelot, P., 2018, PhD thesis, Université Paris-Saclay (ComUE).

[3] Chiodi, R., Desjardins, O., 2017, Journal of Computational Physics, 343, pp. 186-200.

[4] Chorin, A.J., 1967, Journal of Computational Physics, 2, pp. 12-26.

[5] Fedkiw, R.P., Aslam, T., Merriman, B., Osher, S., 1999, Journal of Computational Physics, 152, pp. 457-492.

[6] Gibou, F., Chen, L., Nguyen, D., Banerjee, S. 2007, Journal of Computational Physics, 222, pp. 536-555.

[7] Huh, C., Scriven, L.E., 1971, Journal of Colloid and Interface Science, 35, pp. 85-101.

[8] Janodet, R., Vaudor, G., Lartigue, G., Benard, P., Moureau, V., Mercier, R., Sep. 2.-4. 2019, 29th European Conference on Liquid Atomization and Spray Systems.

[9] Lee, M.S., Riaz, A., Aute, V., 2017, Journal of Computational Physics, 344, pp. 381-418.

[10] Leparoux, J., Mercier, R., Moureau, V., Musaefendic, H., July 22.-26. 2018, 14th Triennal International Conference on Liquid Atomization and Spray Systems.

[11] Moureau, V., Domingo, P., Vervisch, L., 2011, Comptes Rendus Mecanique, 339, pp. 141148.

[12] Olsson, E., Kreiss, G., 2005, Journal of Computational Physics, 210, pp. 225-246.

[13] Sahut, G., 2019, PhD thesis, Université de Grenoble.

[14] Sahut, G., Ghigliotti, G., Balarac, G., Bernard, M., Moureau, V., Marty, P., 2021, Journal of Computational Physics, 432, 110161.

[15] Scriven, L.E., 1959, Chemical Engineering Science, 10, pp. 1-13.

[16] Tanguy, S., Sagan, M., Lalanne, B., Couderc, F., Colin, C., 2014, Journal of Computational Physics, 264, pp. 1-22.

[17] Wang, S., Desjardins, O., 2018, International Journal of Multiphase Flow, 101, pp. 35-46.

[18] Zhang, J., Ni, M.J., 2018, Journal of Computational Physics, 375, pp. 717-746. 